\title{
XLVIII. On the admissible width of the slit in interference experiments
}

\section{James Walker M.A.}

To cite this article: James Walker M.A. (1898) XLVIII. On the admissible width of the slit in interference experiments, Philosophical Magazine Series 5, 46:282, 472-478, DOI: $10.1080 / 14786449808621219$

To link to this article: http://dx.doi.org/10.1080/14786449808621219

曲 Published online: 08 May 2009.

Submit your article to this journal $[\pi$

Џ Article views: 2

Q View related articles $\asymp$ 
mentally by employing very dilute solutions and a large accumulator-battery, such as that recently employed by Professors Trowbridge and Richards*.

20 th May, 1898.

Note added May 27th.-Dr. Van Everdingen informs me that he has discovered the slip in his calculation, and agrees with the remarks made above concerning the experiments of Florio and Bagard.

XLVIII. On the Admissible Width of the Slit in Interference Experiments. By James W alker, M.A. $\dagger$

1. TN a paper published in the Philosophical Magazine for 1 August 1889, Lord Rayleigh has discussed the question of the admissible width of the slit forming the proximate source of light in interference experiments, and has pointed out that "In Fresnel's experiment, whether carried out with mirrors or with biprism ... the condition for distinctness is simply that the width of the slit be a small proportion of the width of the bands $f . "$ This rule, which applies to the case in which the variation of the width of the bands is produced by a change in the mirrors or biprism, the distances of the slit and screen of observation from the apparatus remaining constant, requires modification when it is the variation of the distances that causes the alteration of the width of the bands, the apparatus remaining otherwise unchanged ; for it is an experimental fact that in this latter case, as the bands become finer, the slit may be made wider without loss of distinctness. A more detailed investigation leads to this result, and shows moreover that a progressive widening of the slit causes a periodic disappearance of the bands, the system of bands at the successive reappearances being alternately bright and dark centred §.

2. We require first the relative retardation at a point of the screen of observation of the streams emanating from any point of the slit.

Fresnel's Mirrors.-Let the line of intersection of the mirrors be the axis of $y$, and let the axis of $z$ be taken so as to pass through the image of the centre of the slit in the

* Phil. Mag, February (1897).

† Communicated by the Author.

f Phil. Mag. [5] xrviii. p. 80 (1889).

$\S$ I find that this result of the progressive widening of the slit has already been given by M. Ch. Fabry in his thesis for the degree of Doctor of Science, published at Marseilles in 1892, and regret that I was not aware of this interesting work until my paper was in type. 
plane bisecting the acute angle $2 \omega$ between the mirrors; then the equations of the planes of the mirrors may be written

$$
\begin{aligned}
& x \sin (\theta-\omega)+z \cos (\theta-\omega)=0, \\
& x \sin (\theta+\omega)+z \cos (\theta+\omega)=0 .
\end{aligned}
$$

Suppose the slit perpendicular to the plane through the centre of the slit and the line of intarsection of the mirrors, it edges being perpendicular to the plane of $x z$, and let $a$ be the distance of the central line of the slit from the axis of $y$. The coordinates of a point of the slit distant $\xi$ from this central line are

$$
x_{0}=a \sin 2 \theta+\xi \cos 2 \theta, \quad y_{0}, \quad z_{0}=a \cos 2 \theta-\xi \sin 2 \theta,
$$

and the coordinates of its inage in the first mirror are

$x_{0}-2 \sin (\theta-\omega)\left\{x_{0} \sin (\theta-\omega)+z_{0} \cos (\theta-\omega)\right\}=\iota \sin 2 \omega+\xi \cos 2 \omega$,

$y_{0}$,

$z_{0}-2 \cos (\theta-\omega)\left\{x_{0} \sin (\theta-\omega)+z_{0} \cos (\theta-\omega)\right\}=-a \cos 2 \omega+\xi \sin 2 \omega$.

Hence, the propagational speed of light being taken as unity, the undulatory time of passage, from the point $x_{0}, y_{0}, z_{0}$ to the point $x, y, b$, of the stream reflected at this mirror is

$$
\begin{aligned}
& \mathrm{V}_{1}=\left\{(x-a \sin 2 \omega-\xi \cos 2 \omega)^{2}+\left(y-y_{0}\right)^{2}+(b+a \cos 2 \omega-\xi \sin 2 \omega)^{2}\right\}^{\frac{1}{2}} \\
&=\left\{(b+a \cos 2 \omega)^{2}-2(x \cos 2 \omega+b \sin 2 \omega) \xi+(x-a \sin 2 \omega)^{2}\right. \\
&\left.+\left(y-y_{0}\right)^{2}+\xi^{2}\right\}^{\frac{1}{2}} \\
&= b+u \cos 2 \omega-\frac{x \cos 2 \omega+b \sin 2 \omega}{l+a \cos 2 \omega} \xi+\frac{1}{2}(x-a \sin 2 \omega)^{2}+\left(y-y_{0}\right)^{2}+\xi^{2} \\
& b+a \cos 2 \omega
\end{aligned}
$$

The undulatory time of passag ${ }_{3} \mathrm{~V}_{2}$ between the same two points of the stream reflected at the second mirror is obtained from $V_{1}$ by changing the sign of $\omega$; hence the relative retardation, measured in length in air, is

$$
\Delta=V_{2}-V_{1}=2 \frac{\sin 2 \omega(b \xi+a x)}{b+a \cos 2 \omega} .
$$

Fresnel's Biprism.-Let the plane through the edge of the prism perpendicular to the flat face be the plane of $y z$, the elge being parallel to the axis of $y$, and suppose that the flat face is turned towards the slit, which is placed in the plane $z=0$ with its central line along the axis of $y$.

If $a$ be the distance of the edge of the prism from the origin, and $\alpha_{1}, \alpha_{2}$ be the acute angles of the prism, the equations to its inclined faces will be

$$
\begin{aligned}
& z=a-\tan \alpha_{1} x, \\
& z=a+\tan \alpha_{2} x .
\end{aligned}
$$


Let $t$ be the distance of the flat face of the prism from its edge, and $z=a+b$ the equation to the screen of observation, then if the ray from the point $\xi, \eta$ of the slit to the point $x, y$ of the screen meet the faces of the half of the prism on the side of positive $x$ in the points $x_{1}, y_{1}, a-t$ and $x_{2}, y_{2}, z_{2}$ respectively, the undulatory time of passage of the light that passes through this half of the prism is

$$
\begin{aligned}
& \mathrm{V}_{1}=\left\{\left(x_{1}-\xi\right)^{2}+\left(y_{1}-\eta\right)^{2}+(a-t)^{2}\right\}^{\frac{1}{2}}+\mu\left\{\left(x_{2}-x_{1}\right)^{2}+\left(y_{2}-y_{1}\right)^{2}+\left(z_{2}-a+t\right)^{2}\right\}^{\frac{1}{2}} \\
& +\left\{\left(x-x_{2}\right)^{2}+\left(y-y_{2}\right)^{2}+\left(a+b-z_{2}\right)^{2}\right\}^{\frac{2}{2}} \\
& \doteqdot a-t+\mu\left(z_{2}-a+t\right)+\left(a+b-z_{2}\right) \\
& +\frac{1}{2}\left\{\frac{\left(x_{1}-\xi\right)^{2}+\left(y_{1}-\eta\right)^{2}}{a-t}+\mu \frac{\left(x_{2}-x_{1}\right)^{2}+\left(y_{2}-y_{1}\right)^{2}}{z_{2}-a+t}\right. \\
& \left.+\frac{\left(x-x_{2}\right)^{2}+\left(y-y_{2}\right)^{2}}{a+b-z_{2}}\right\} \\
& \doteqdot a+b+(\mu-1) t-(\mu-1) \tan \alpha_{1} \cdot x_{2} \\
& +\frac{1}{2}\left\{\frac{\left(x_{1}-\xi\right)^{2}+\left(y_{1}-\eta\right)^{2}}{a-t}+\mu \frac{\left(x_{2}-x_{1}\right)^{2}+\left(y_{2}-y_{1}\right)^{2}}{t}\right. \\
& \left.+\frac{\left(x-x_{2}\right)^{2}+\left(y-y_{2}\right)^{2}}{b}\right\}
\end{aligned}
$$

with the conditions

whence

$$
\begin{aligned}
& \frac{d \mathrm{~V}_{1}}{d x_{1}}=\frac{x_{1}-\xi}{a-t}+\mu \frac{x_{1}-x_{2}}{t}=0, \\
& \frac{d \mathrm{~V}}{d y_{1}}=\frac{y_{1}-\eta}{a-t}+\mu \frac{y_{1}-y_{2}}{t}=0, \\
& \frac{d \Gamma_{1}}{d x_{2}}=-(\mu-1) \tan x_{1}-\mu \frac{x_{1}-x_{2}}{t}+\frac{x_{2}-x}{b}=0, \\
& \frac{d \mathrm{~V}}{d y_{2}}=-\mu \frac{y_{1}-y_{2}}{t}+\frac{y_{2}-y}{b}=0 ;
\end{aligned}
$$

$$
\begin{aligned}
V_{1}= & a+b+(\mu-1) t-(\mu-1) \tan \alpha_{1} \cdot x_{2} \\
& +\frac{1}{2}\left[\left\{\frac{\mu^{2}(a-t)}{t^{2}}+\frac{\mu}{t}\right\}\left(x_{1}-x_{2}\right)^{2}+b\left\{(\mu-1) \tan \alpha_{1}+\frac{\mu}{t}\left(x_{1}-x_{2}\right)\right\}^{2}\right] \\
& +\frac{1}{2}\left\{\frac{\mu^{2}(a-t)}{t^{2}}+\frac{\mu}{t}+\frac{\mu^{2} b}{t^{2}}\right\}\left(y_{1}-y_{2}\right)^{2} \\
= & a+b+(\mu-1) t+\frac{1}{2} b(\mu-1)^{2} \tan ^{2} \alpha_{1}+\frac{\mu}{t} b(\mu-1) \tan \alpha_{1} \cdot\left(x_{1}-x_{2}\right) \\
- & (\mu-1) \tan \alpha_{1} \cdot x_{2}+\frac{1}{2} \cdot \frac{\mu}{t^{2}}\{\mu(a+b)-(\mu-1) t\}\left\{\left(x_{1}-x_{2}\right)^{2}+\left(y_{1}-y_{2}\right)^{2}\right\} .
\end{aligned}
$$


But

$$
\begin{aligned}
& x_{2}=\xi-\frac{\xi-x-(\mu-1) b \tan \alpha_{1}}{\mu(a+b)-(\mu-1) t}\{\mu(a-t)+t\}, \\
& x_{1}-x_{2}=\frac{\xi-x-(\mu-1) b \tan \alpha_{1}}{\mu(a+b)-(\mu-1) t} \cdot t, \\
& y_{1}-y_{2}=\frac{\eta-y}{\mu(a+b)-(\mu-1) t} \cdot t ; \\
& \therefore \quad \nabla_{1}=a+b+(\mu-1) t+\frac{1}{2} \cdot b(\mu-1)^{2} \tan ^{2} \alpha_{1} \\
& \quad+\mu b(\mu-1) \tan \alpha_{1} \cdot \frac{\xi-x-(\mu-1) b \tan \alpha_{1}}{\mu(a+b)-(\mu-1) t} \\
&-(\mu-1) \tan \alpha_{1}\left[\xi-\frac{\xi-x-(\mu-1) b \tan \alpha_{1}}{\mu(a+b)-(\mu-1) t}\{\mu a-(\mu-1) t\} !\right. \\
& \quad+\frac{\mu}{2} \frac{\left\{\xi-x-(\mu-1) b \tan \alpha_{1}\right\}^{2}+(\eta-y)^{2},}{\mu(a+b)-(\mu-1) t}
\end{aligned}
$$

which becomes on reduction

$$
\begin{aligned}
\nabla_{1}= & a+b+(\mu-1) t-\frac{1}{2} \cdot \frac{b\{\mu a-(\mu-1) t\}(\mu-1)^{2} \tan ^{2} \alpha_{1}}{\mu(a+b)-(\mu-1) t} \\
& +\frac{\mu}{2} \frac{\xi^{2}+\eta^{2}+x^{2}+y^{2}}{\mu(a+b)-(\mu-1) t}-\mu \cdot \frac{\eta y}{\mu(a+b)-(\mu-1) t} \\
& -\frac{\left.\mu \xi x+(\mu-1) \tan \alpha_{1}[\mu b) \xi+\{\mu a-(\mu-1) t\}, v\right]}{\mu(a+b)-(\mu-1) t} .
\end{aligned}
$$

The undulatory time of passage $V_{2}$ between the same two points of the stream that passes through the other half of the prism is obtained from $V_{1}$ by writing $-\tan \alpha_{2}$ for $\tan \alpha_{1}$; hence the relative retardation of the streams, measured in length in air, is

$$
\begin{aligned}
\Delta=\mathrm{V}_{2}-\mathrm{V}_{1}= & \frac{1}{2} \cdot \frac{b\{\mu a-(\mu-1) t\}(\mu-1)^{2}\left(\tan ^{2} \alpha_{1}-\tan ^{2} \alpha_{2}\right)}{\mu(a+b)-(\mu-1) t} \\
& +\frac{\left.(\mu-1)\left(\tan \alpha_{1}+\tan \alpha_{2}\right)[\mu b)+\{\mu a-(\mu-1) t\} x\right]}{\mu(a+b)-(\mu-1) t} .
\end{aligned}
$$

Billet's Divided Lens.-Suppose the slit placed symmetrically with respect to the two halves of the lens in a direction perpendicular to the plane through their principal axes : then an investigation similar to the above leads to the result that at the point $x, y$ of the screen of observation, the relative retardation of the two streams that emanate from the 
point $\xi, \eta$ of the slit and traverse each one half of the lens is

$$
\Delta=2 \epsilon \cdot \frac{\left(b-t+\frac{t}{\mu} \cdot \frac{\mu-1}{r_{1}} \mathrm{~F}\right) \xi+\left(a+\frac{t}{\mu} \cdot \frac{\mu-1}{r_{2}} \mathrm{~F}\right) x}{a(b-t)-\mathrm{F}(a+b-t)-\mathrm{F} \frac{t}{\mu}\left\{1-\frac{\mu-1}{r_{1}} a-\frac{\mu-1}{r_{2}}(b-t)\right\}}
$$

where $2 \epsilon$ is the separation of the halves of the lens,

$t$ is the thickness of the lens,

$a, b$ are the distances, measured along the axis, of the slit and the screen from the surface of the lens nearest the slit,

$r_{1}, r_{2}, F$ are the absolute values of the radii of the surfaces and of the focal length of the lens.

3. In each of these three cases the intensity at a point of the screen due to an element of the slit of breadth $d \xi$ distant $\xi$ from its central line may be written

$$
h\left\{1+\cos \frac{2 \pi}{\lambda}(\alpha+\beta x+\gamma \xi)\right\} d \xi,
$$

where $\alpha, \beta$, and $\gamma$ have the values proper to the case under consideration ; and if the various parts of the width of the slit act as independent sources of light-the condition, as Lord Rayleigh has shown (lor. cit. p. 81), most favourable to brightness - the intensity due to the whole slit of width $k$ is

$$
\begin{aligned}
I & =h \int_{-k ; 2}^{k / 2}\left\{1+\cos \frac{2 \pi}{\lambda}(\alpha+\beta x+\gamma \xi)\right\} d \xi \\
& =h k\left\{1+\frac{\sin \pi \gamma k / \lambda}{\pi \gamma k / \lambda} \cos \frac{2 \pi}{\lambda}(\alpha+\beta x)\right\} .
\end{aligned}
$$

The visibility of the interference-fringes is thus given by $\pm \sin (\pi \gamma k / \lambda) /(\pi \gamma k / \lambda)$; this vanishes when $\gamma k / \lambda$ is an integer, while the maxima of distinctness occur when

or when

$$
\tan (\pi \gamma k / \lambda)=\pi \gamma k / \lambda
$$

$$
\gamma k / \gamma=0, \quad 1 \cdot 4303, \quad 2 \cdot 4590, \quad 3 \cdot 4709, \quad 4 \cdot 4747, \ldots,
$$

the corresponding values of the visibility being

$$
1 \quad \cdot 217 \quad .128 \quad \cdot 091 \quad \cdot 079 \ldots \text {; }
$$

when $\gamma / 2 / \lambda=0$, the intensity is zero, but the visibility will be considerable so long as $\gamma k / \lambda$ is small. Now the linear width of the bands (from bright to bright, or from dark to dark) being

$$
\Lambda=\lambda / \beta,
$$

the condition for maximum distinctness is that $k$ must be a 
small fraction of $\beta \Lambda / \gamma$, and referring to the results obtained above, we see that in the case of Fresnel's mirrors, and in the cases of the biprism and of the divided lens when the thickness is neglected, $\beta / \gamma=a / b$, so that the condition for maximum distinctness is that the width of the slit must be a small fraction of $a / b$ times the width of the bands, where $a$ and $b$ are the distances of the interference apparatus from the slit and screen of observation respectively*.

In the case of the biprism when its thickness is neglected, if $a$ and $b$ alone vary, their sum remaining constant, $a \Lambda$ is constant, and hence, in order that the distinctness may remain unchanged, the width of the slit must be inversely as $b$, that is the narrower the bands, the greater the admissible width of the slit.

Starting from the case of maximum distinctness and gradually increasing the width of the slit, we see that the interference-bands will become less and less distinct; they then vanish and reappear again in the complementary position, since $\sin (\pi \gamma k / \lambda) /(\pi \gamma k / \lambda)$ changes sign on passing through the value zero, and increase in distinctness up to a maximun which is about a fifth of the prime maximum of distinctness, and so on.

An interesting method of observing this phenomenon is to allow white light to pass and to subsequently analyse the mixture by a spectroscope with its slit placed at right angles to the interference-bands. When the source of light is a narrow slit, the ordinary fan-like appearance is obtained, that is a spectrum traversed by slightly curred bands running more or less along the spectrum and approaching one another towards the violet end. As the source of light is gradually made wider the bands become less distinct, the distinctness decreasing most rapidly at the violet end, until a region with no bands takes its rise at that end and passes along the spectrum to the red end, to be followed by a second such region and so on, the bands on the two sides of the bandless region being complementary.

This method also affords a means of determining the "limit of visibility" for a given wave-length; for by using a micrometer slit as the proximate source of light and aljusting its width until one of the limits of the bandless region coincides with a given Fraunhofer line, the quantities that occur in the expression for the visibility may be determined

* In other words, the angle subtended by the slit at the interference apparatus must be a small proportion of that subtended by the width of the bands at the same point, a result that might be arrived at by elementary reasoning.

Phil. Mag. S. 5. Vol. 46. No. 282. Nov. 1898. $2 \mathrm{~L}$ 
and the limit of visibility for that wave-length may be calculated. In most cases, however, diffraction complicates the phenomenon, and if great accuracy be required, a more complete investigation would be necessary.

4. Lloyd's Bands.-The case of Llnyd's Bands is slightly different (Lord Rayleigh, loc. cit. p. 80). If $\xi$ be the distance of the luminous point from the plane of the mirror, the relative retardation of the reflected and direct streams at a point of the screen distant $x$ from its line of intersection with the plane of the mirror is (measured in length) $2 x \xi / d$, where $d$ is the distance of the luminous point from the screen, supposed at right angles to the plane of the mirror.

The central line of the slit being at a distance $c$ from the plane of the mirror, the intensity due to the whole slit of width $k$ may be written

$$
\begin{aligned}
\mathrm{I} & =h \int_{c-k / 2}^{c+k_{i} / 2}\left\{1+\cos \frac{2 \pi}{\lambda} \cdot \frac{2 x \xi}{d}\right\} d \xi \\
& =h k\left\{1+\frac{\sin \frac{2 \pi}{\lambda} \cdot \frac{x k}{d}}{\frac{2 \pi}{\lambda} \cdot \frac{x k}{d}} \cdot \cos \frac{2 \pi}{\lambda} \cdot \frac{2 c x}{d}\right\} .
\end{aligned}
$$

The visibility is here given by $\pm \sin (\pi n k / c) /(\pi n k / c)$, where $n$ is the order of the bands, and the condition for maximum distinctness is that the width of the slit must be a small fraction of $c / n$, and thus depends upon the order of the bands: in other respects the phenomenon is the same as in the former cases.

This dependence of the visibility on the order of the bands and their periodic disappearance may be observed with homogeneous light by leaving the width of the slit unaltered and moving backwards the oyepiece with which the bands are observed, keeping it all the time in the doubly illuminated field.

XLIX. On the Distribution of Magnetic Induction in Straight Iron Rods. By J. L. W. GILL, B.A.Sc., 1851 Exhibition Scholar*.

DURING the summer and autumn of 1897 the author was engaged in working out the details of a new method of measuring magnetic hysteresis in iron. The fundamental principle of this method is the measurement of the mechanical work expended when a specimen of iron is passed through a magnetic field (see 'Electrician,' Sept. 24, 1897).

\footnotetext{
* Communicated by the Author.
} 